Pacific Journal of Mathematics

SECTIONAL REPRESENTATIONS OF BANACH MODULES

TOn KITCHEN, Jr, and David A Robins 


\title{
SECTIONAL REPRESENTATIONS OF BANACH MODULES
}

\author{
J. W. Kitchen AND D. A. RobBins
}

\begin{abstract}
This paper is concerned with the representation of Banach algebras and Banach modules as sections of bundles of Banach spaces. In particular: (1) if $A$ is a commutative Banach algebra, then $A$ may be represented by sections of a locally trivial canonical line bundle; (2) if $A$ is a Banach algebra which is represented as sections of a canonical bundle of Banach algebras, then there is a natural way in which any Banach module $M$ over $A$ can be represented by sections of a canonical bundle of Banach modules over the corresponding algebra bundle. We also investigate projective tensor products of bundles of Banach algebras and bundles of Banach modules.
\end{abstract}

The present paper extends and generalizes earlier results to be found in [7] and [8], to which the reader is referred for terminology and notations. Both of these papers center upon the study of sectional representations of "Gelfand type" for Banach modules $(M, A)$ such that

(1) the algebra $A$ is commutative, and

(2) the module satisfies the (KR) condition.

Such modules must be essential, and conversely, every essential module over an algebra with bounded approximate identities satisfies the (KR) condition. In the present paper it is shown that the (KR) condition can be dropped altogether provided one is willing to accept a somewhat altered version of the classical Gelfand representation of $A$. Rather than represent $A$ by complex-valued functions, one can represent $A$ by sections of a canonical line bundle. This "primitive" Gelfand representation is described in $\S 1$, which concerns bundles of Banach algebras more generally.

$\$ 2$ concerns sectional representation of Banach modules. A new construction for canonical bundles of Banach modules is given (Theorem 2.3), which generalizes various known constructions. Basically, Theorem 2.3 states that whenever one has a representation of a (possibly noncommutative) Banach algebra $A$ by sections of a canonical algebra bundle, then there is a natural means whereby one can represent every module $M$ over $A$, namely, $M$ can be represented by sections of a canonical bundle of Banach modules over the corresponding algebra bundle. This result applies, in particular, to the primitive Gelfand representation of a commutative algebra. It applies also to the sectional representations of $C^{*}$-algebras which have been studied by Hofmann, Varela, Dupré, et al. Moreover, the 
closing portion of $\$ 2$ makes clear the true role of the $(\mathrm{KR})$ condition (Theorem 2.6).

The third section of the paper treats projective tensor products of bundles and their relationships to the sectional representations studied in $\S \S 1$ and 2.

\section{Bundles of Banach algebras, the Gelfand representation revisited.} We turn first to the representation of Banach algebras by sections of bundles of Banach algebras.

Suppose that $\pi: E \rightarrow S$ is a bundle of Banach spaces in which the stalks $E_{p}=\pi^{-1}(p), p \in S$, are all Banach algebras. Let us denote by $m$ the map from the fibered product $E \vee E=\{(x, y) \in E \times: \pi(x)=\pi(y)\}$ into $E$ which assigns to any pair $(x, y)$ in $E \vee E$ the product $x y$ in the algebra $E_{\pi(x)}=E_{\pi(y)}$.

Proposition 1.1. Let $\pi: E \rightarrow S$ be as above. The following are equivalent:

(1) the map $m: E \vee E \rightarrow E$ is continuous;

(2) given any two elements $x_{0}$ and $y_{0}$ belonging to the same stalk, and given any neighborhood $\mathcal{Q}$ of $x_{0} y_{0}$, there exist neighborhoods $\mathcal{Q}$ and $\mathcal{V}$ of $x_{0}$ and $y_{0}$, respectively, such that $x y \in \mathcal{Q} \int$ whenever $x \in \mathcal{Q}, y \in \mathcal{V}$ and $\pi(x)=$ $\pi(y)$;

(3) the local sections of the bundle are closed under pointwise multiplication;

(4) There exists a full set $\mathbb{Q}$ of local sections which is closed under pointwise multiplication.

When the conditions in Proposition 1.1 are satisfied $\pi: E \rightarrow S$ is called a bundle of Banach algebras. Such bundles have been defined for quite some time in terms of condition (1). Because of (3) it follows that the set $\Gamma(\pi)$ of all global sections of such a bundle forms an algebra with respect to pointwise operations. Moreover, the space $\Gamma_{b}(\pi)$ of all bounded sections of such a bundle is a Banach algebra (using, of course, the sup norm $\|\sigma\|=\sup \{\|\sigma(p)\|: p \in S\})$. The main usefulness of Proposition 1.1 comes, however, from the fact that (4) implies (1). To specialists in Banach bundles this result will probably come as no surprise. What is surprising, according to the referee, is that the equivalence of (1) and (4) seems to be nowhere on record.

The proof of Proposition 1.1 is routine and is therefore omitted. It should be mentioned, however, that in the absence of further assumptions 
concerning $S$ (that it is completely regular Hausdorff, for instance), it must be clearly understood exactly what definition of bundle is being employed. In this paper, as in [7] and [8], it is assumed that every point in the fibre space of a bundle of Banach spaces lies on a local section of the bundle, i.e., that the set of all local sections is full. (In [6] a weaker requirement is made.)

This brings us to the central topic of this section: the representation of Banach algebras by sections of bundles of Banach algebras. Thus, given a Banach algebra $A$ we shall be interested in constructing algebra homomorphisms $\phi: A \rightarrow \Gamma_{b}(\pi)$ where $\pi: E \rightarrow S$ is a bundle of Banach algebras. The standard technique for constructing sectional representations of $A$ is described in our next theorem, which is "a part of the established folklore," to quote the referee once again.

If $X$ is a Banach space and $\left\{X_{p}: p \in S\right\}$ is a collection of closed subspaces of $X$ indexed by the topological space $S$, then $\left\{X_{p}: p \in S\right\}$ is said to be topologically compatible with $S$ provided that the function $p \mapsto\left\|x+X_{p}\right\|$ is upper semicontinuous for each $x \in X$. (See [7].)

Proposition 1.2. (Existence of Canonical Bundles of Banach Algebras.) Let $A$ be a Banach algebra, let $S$ be a topological space, and suppose that $\left\{I_{p}: p \in S\right\}$ is a family of closed two-sided ideals in $A$ which is topologically compatible with $S$. Then the canonical bundle of Banach spaces $\pi: E \rightarrow S$ defined by the family $\left\{I_{p}: p \in S\right\}$ is a bundle of Banach algebras and the associated Gelfand morphism ^ $: A \rightarrow \Gamma_{b}(\pi)$ is a norm descreasing representation of $A$.

Proof. In the canonical bundle $\pi: E \rightarrow S$ the stalk above a point $p \in S$ is (isomorphic to) the quotient space $A / I_{p}$, which is a Banach algebra. If $a \in A$ and $p \in S$, then $\hat{a}(p)$ is (can be identified with) the coset $a+I_{p}$. Clearly,

$$
\widehat{a b}(p)=a b+I_{p}=\left(a+I_{p}\right)\left(b+I_{p}\right)\left(b+I_{p}\right)=\hat{a}(p) \hat{b}(p),
$$

for all $a, b \in A$ and $p \in S$; thus, $\widehat{a b}=\hat{a} \hat{b}$. It follows that $\hat{A}=\{\hat{a}: a \in A\}$ is a full set of sections of $\pi$ which is closed under pointwise multiplication. By Proposition 1.1, $\pi: E \rightarrow S$ is a bundle of Banach algebras, etc.

In connection with the preceding result it is appropriate to ask whether every algebra bundle arises as a canonical bundle. For algebra 
bundles with locally compact bases the answer is "yes." Suppose, specifically, that $\pi: E \rightarrow S$ is a bundle of Banach algebras where $S$ is locally compact Hausdorff. We let $A$ be the Banach algebra $\Gamma_{0}(\pi)$ consisting of the sections of $\pi$ which vanish at infinity, and for each $p \in S$ we let $I_{p}$ be the kernel of the evaluation homomorphism $\mathrm{ev}_{p}: A \rightarrow E_{p}$ defined by $\operatorname{ev}_{p}(\sigma)=\sigma(p)$. Then $I_{p}$ is a closed two-sided ideal in $A$, the family $\left\{I_{p}\right.$ : $p \in S\}$ is topologically compatible with $S$, and the associated canonical bundle is bundle isomorphic to the given bundle $\pi: E \rightarrow S$ (by Theorem 3.1 of [7]), etc.

There are a number of important situations in which the preceding theorem applies. We will presently show that for a commutative Banach algebra $A$ the family of maximal regular ideals is compatible with the topology of the maximal ideal space (Corollary 1.6). The corresponding representation will be called the "primitive Gelfand representation of $A$ ", in many important cases it is, for all intents and purposes, the usual Gelfand representation of $A$.

Another important situation where Proposition 1.2 applies is that in which $A$ is a (possibly non-commutative) $C^{*}$-algebra. By a primitive ideal in $A$ we mean, in this case, the kernel of an irreducible Hilbert space representation of $A$. The space $\operatorname{Prim}(A)$ of all primitive ideals in $A$ is given the hull-kernel or Jacobson topology. Unfortunately, $\operatorname{Prim}(A)$ need not be Hausdorff, but when it is, $\{I: I \in \operatorname{Prim}(A)\}$ is topologically compatible with $\operatorname{Prim}(A)$, the associated canonical bundle $\xi: E \rightarrow \operatorname{Prim}(A)$ is a bundle of $C^{*}$-algebras (whose definition should be obvious), and the corresponding Gelfand morphism $\wedge: A \rightarrow \Gamma_{0}(\xi)$ is an isometric isomorphism. When $\operatorname{Prim}(A)$ fails to be Hausdorff, there are several other sectional representations which can be considered. One of them, which is due to J. M. G. Fell [4], will now be described. For the base space we take $\mathscr{T}(A)$, the set of all closed two-sided ideals in $A$. Then $\Phi(A)$ is a compact Hausdorff space when given the topology of pointwise convergence of $C^{*}$-seminorm functions on $A$, where we associate with an ideal $I \in \mathscr{G}(A)$ the seminorm induced on $A$ by the $C^{*}$-algebra norm on the quotient $A / I$. Moreover, $\mathscr{G}(A)$ is actually a compact semigroup under the operation $i$ :

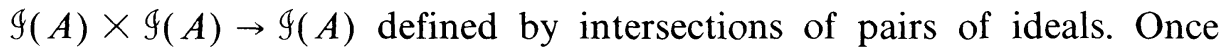
again, the family $\mathscr{T}(A)$ satisfies the hypotheses of Proposition 1.2. For a discussion of the associated representation of $A$ the reader is referred to [3]. More generally, the reader may consult [2] for further work on sectional representations of $C^{*}$-algebras.

We consider next, as promised, the primitive Gelfand representation of a commutative Banach algebra. 
Lemma 1.3. Suppose that $X$ is a Banach space and that $N$ is the kernel of some non-zero functional $f \in X^{*}$. Then, for all $x \in X$,

$$
\|x+N\|=\frac{1}{\|f\|}|f(x)| \text {. }
$$

The proof is left to the reader.

LEMMA 1.4. If $X$ is a Banach space, then the norm function on $X^{*}$ is weakly-* lower semicontinuous.

Proof. The following must be shown: given and $F_{0} \in X^{*}$ with $\left\|F_{0}\right\|>$ $\varepsilon>0$, the inequality $\|F\|>\varepsilon$ holds for all $F$ in some weak-* neighborhood of $F_{0}$. We can, of course, select an element $x_{0} \in X$ of norm one such that $\left|F_{0}\left(x_{0}\right)\right|>\varepsilon$. The set $V=\left\{F \in X^{*}:\left|F\left(x_{0}\right)\right|>\varepsilon\right\}$ is an open weak-* neighborhood of $F_{0}$, and, for all $F \in V,\|F\|>\varepsilon$.

Proposition 1.5. Let $X$ be a Banach space and let $S$ be a set of non-zero functionals in $X^{*}$. For each $f \in S$, let $N_{f}$ be the kernel of $f$. Then, if $S$ is given the weak-* topology, the family $\left\{N_{f}: f \in S\right\}$ is topologically compatible with $S$.

Proof. We must show that for each $x \in X$, the map $f \rightarrow\left\|x+N_{f}\right\|$ is upper semicontinuous on $S$. But

$$
\left\|x+N_{f}\right\|=\frac{1}{\|f\|}|f(x)| .
$$

The map $f \rightarrow f(x)$ is, of course, weakly-* continuous. Since the norm function is weak-* lower semicontinuous on $X^{*}$, it follows that the map $f \rightarrow 1 /\|f\|$ is weakly-* upper semicontinuous on $S$. Thus, the map $f \rightarrow$ $\left\|x+N_{f}\right\|$ is upper semicontinuous on $S$.

COROLlary 1.6. Let $A$ be a commutative Banach algebra with maximal ideal space $\Delta$. For each $h \in \Delta$ let $I_{h}$ be the corresponding regular maximal ideal in $A$. Then the family $\left\{I_{h}: h \in \Delta\right\}$ is topologically compatible with $\Delta$.

Proof. View the elements of $\Delta$ as multiplicative functionals.

We can now apply the previous corollary together with Proposition 1.2. Let $A$ be a commutative Banach algebra with maximal ideal space $\Delta$. Let $\pi: E \rightarrow \Delta$ be the canonical bundle corresponding to the family $\left\{I_{h}\right.$ : $h \in \Delta\}$ and let $\sim: A \rightarrow \Gamma_{b}(\pi)$ be the corresponding Gelfand morphism. 
Thus, as a set $E$ may be written:

$$
E=\left\{\left(h, a+I_{h}\right): h \in \Delta, a \in A\right\}
$$

and for $a \in A$ and $h \in \Delta$

$$
\tilde{a}(h)=\left(h, a+I_{h}\right) .
$$

The map $\sim: A \rightarrow \Gamma_{b}(\pi)$ will be called the primitive Gelfand representation of $A$. Its relation to the usual Gelfand representation: $A \rightarrow C_{0}(\Delta)$ can be described as follows. Let $\gamma: \Delta \times \mathbf{C} \rightarrow \Delta$ be the constant bundle, that is, $\Delta \times \mathbf{C}$ has the product topology, $\gamma$ is the natural coordinate projection, and $\|(h, \lambda)\|=|\lambda|$ for all $h \in \Delta$ and $\lambda \in \mathbf{C}$. Then there is a well-defined bijective map $\phi: E \rightarrow \Delta \times \mathbf{C}$ such that

$$
\phi\left(\left(h, a+I_{h}\right)\right)=(h, \hat{a}(h))
$$

for all $a \in A$ and $h \in \Delta$. It can then be shown that $\phi$ is a norm-decreasing bundle map or morphism in the category of bundles of Banach algebras. That is, $\phi$ is not only a bundle map when $\pi: E \rightarrow \Delta$ and $\gamma: \Delta \times \mathbf{C} \rightarrow \Delta$ are viewed as bundles of Banach spaces, but, in addition, the restricted mappings of stalks $\phi_{h}: E_{h} \rightarrow(\Delta \times \mathbf{C})_{h}$ are algebra homomorphisms. If, finally, we denote by $\Phi: \Gamma_{b}(\gamma) \rightarrow C_{b}(\Delta)$ the natural way of identifying sections of the constant bundle with continuous complex-valued functions on $\Delta$, then the two Gelfand representations are related by the equation:

$$
\hat{x}=\Phi(\phi \circ \tilde{x}),
$$

for all $x \in A$.

Proposition 1.7. Given any commutative Banach algebra $A$ there exists a bijective bundle map $\phi$ of norm one or less from the canonical bundle $\pi: E \rightarrow \Delta$ to the constant bundle $\gamma: \Delta \times \mathbf{C} \rightarrow \Delta$ such that

$$
\hat{x}=\Phi(\phi \circ \tilde{x}),
$$

for all $x \in A$. If $A$ has an identity with norm one or a bounded approximate identity all of whose elements have norm less than or equal to one, then $\phi$ is an isometric homeomorphism.

Let us pursue the present discussion a bit further with regard to identities. If $A$ has an identity $e$ then $\tilde{e}$ is the identity section $\sigma_{e}$ which assigns to each $h \in \Delta$ the identity coset of $E_{h} \simeq A / I_{h}$; furthermore $\sigma_{e}$ is the identity element of the algebra $\Gamma_{b}(\pi)$. If $A$ lacks an identity then the selection $\sigma_{e}: \Delta \rightarrow E$ need not belong to $\tilde{A}=\{\tilde{a}: a \in A\}$ nor even to $\Gamma_{b}(\pi)$. It is, however, a global section. 
Proposition 1.8. If $A$ is a commutative Banach algebra, then the identity selection $\sigma_{e}: \Delta \rightarrow E$ defined above is a (possibly unbounded) section of the canonical bundle $\pi: E \rightarrow \Delta$.

Proof. Let us prove continuity of $\sigma_{e}$ at an arbitrary point $h_{0} \in \Delta$. Let $u_{0}$ be an identity for $A$ modulo $I_{h_{0}}$. Thus $\tilde{u}_{0}\left(h_{0}\right)=\sigma_{e}\left(h_{0}\right)$ and $\hat{u}_{0}\left(h_{0}\right)=1$. Let $V$ be any neighborhood of $\sigma_{e}\left(h_{0}\right)$. We may assume that $\mathcal{V}$ is of the form

$$
\mathscr{V}=\left\{z \in E: \pi(z) \in U,\left\|z-\tilde{u}_{0}(\pi(z))\right\|<\varepsilon\right\}
$$

where $U$ is a neighborhood of $h_{0}$ having compact closure in $\Delta$. Since the function $h \rightarrow 1 /\|h\|$ is upper semicontinuous on $\bar{U}$ it attains a maximum value there, say $M$. Because of the continuity of the complex-valued function $\hat{u}_{0}$ we can find a neighborhood $V \subset U$ of $h_{0}$ such that

$$
h \in V \Rightarrow\left|\hat{u}_{0}(h)-1\right|=\left|\hat{u}_{0}(h)-\hat{u}_{0}\left(h_{0}\right)\right|<\varepsilon / M .
$$

Suppose, then, that $h \in V$. Let $u_{h}$ be an identity for $A$ modulo $I_{h}$. Then

$$
\begin{aligned}
\left\|\sigma_{e}(h)-u_{0}(h)\right\| & =\left\|\left(u_{h}-u_{0}\right)(h)\right\| \\
& =\frac{1}{\|h\|}\left|\left(u_{h}-u_{0}\right)^{\wedge}(h)\right|=\frac{1}{\|h\|}\left|1-\hat{u}_{0}(h)\right| \\
& <M \cdot(\varepsilon / M)=\varepsilon .
\end{aligned}
$$

Thus $\sigma_{e}(h) \in V$. This proves that $\sigma_{e}$ is continuous at $h_{0}$.

COROLlary 1.9. If $A$ is a commutative Banach algebra, then the canonical bundle $\pi: E \rightarrow \Delta$ is topologically trivial. Specifically, the bundle map $\phi$ is a homeomorphism of the fibre space $E$ onto $\Delta \times \mathbf{C}$.

Proof. Let $h_{0} \in \Delta$. It suffices to show that $\phi$ maps each neighborhood of $\left(h_{0}, \sigma_{e}\left(h_{0}\right)\right)$ onto a neighborhood of $\phi\left(h_{0}, \sigma_{e}\left(h_{0}\right)\right)=\left(h_{0}, 1\right)$. (Note that the scalar multiples of $\sigma_{e}$ provide a full set of sections of the canonical bundle $\pi: E \rightarrow \Delta$.) Every neighborhood of $\left(h_{0}, \sigma_{e}\left(h_{0}\right)\right)$ contains a neighborhood of the form

$$
\begin{aligned}
V & =\left\{\left(h, \lambda \sigma_{e}(h)\right): h \in U,\left\|(\lambda-1) \sigma_{e}(h)\right\|<\varepsilon\right\} \\
& =\left\{\left(h, \lambda \sigma_{e}(h)\right): h \in U,|\lambda-1|<\varepsilon /\left\|\sigma_{e}(e)\right\|\right\}
\end{aligned}
$$

where $U$ is a compact neighborhood of $h_{0}$ in $\Delta$. The bundle map $\phi$ carries $V$ onto the set

$$
W=\left\{(h, \lambda): h \in U,|\lambda-1|<\varepsilon /\left\|\sigma_{e}(h)\right\|\right\} .
$$


If we let $\eta$ be the minimum value of the lower semicontinuous function $1 /\left\|\sigma_{e}(h)\right\|$ on $U$, then $W$ clearly contains

$$
\{(h, \lambda): h \in U,|\lambda-1|<\varepsilon \eta\},
$$

which is recognizably a neighborhood of $\left(h_{0}, 1\right)$ in $\Delta \times \mathbf{C}$.

It follows from Corollary 1.9 that the difference between the canonical bundle $\pi: E \rightarrow \Delta$ for $A$ and the constant bundle $\gamma: \Delta \times \mathbf{C} \rightarrow \Delta$ lies in the norms as opposed to the topologies of the fibre spaces. We conclude this section with an example which will illustrate the differences. For $A$ we take a modification of the group algebra $L^{1}(\mathbf{T})$, where $\mathbf{T}=\mathbf{R} / \mathbf{Z}$ is the circle group with normalized Haar measure $d t$.

In the case of the group algebra the maximal ideal space can be identified with $\mathbf{Z}$. For each $n \in \mathbf{Z}$ we let $\phi_{n}$ be the $n$th character: $\phi_{n}(t)=e^{2 n \pi i t}$. The corresponding multiplicative functional $h_{n}$ assigns to each $x \in L^{1}(\mathbf{T})$ its $n$th Fourier coefficient $\left(x, \phi_{n}\right)$ where we use the pair notation for the usual duality between $L^{1}$ and $L^{\infty}$, that is, $(f, g)=$ $\int f(t) \overline{g(t)} t$ for all $f \in L^{1}(\mathbf{T})$ and $g \in L^{\infty}(\mathbf{T})$. Thus, if we let $I_{n}=\operatorname{ker} h_{n}$ be the corresponding maximal ideal, then, for all $x \in L^{1}(\mathbf{T})$ and $n \in \mathbf{Z}$,

$$
\left\|x+I_{n}\right\|=\|\tilde{x}(n)\|=\frac{1}{\left\|h_{n}\right\|}|\hat{x}(n)|=\left|\left(x, \phi_{n}\right)\right| .
$$

We now take $A$ to be a modification of $L^{1}(\mathbf{T})$. We select any family of positive numbers $\left\{\nu_{n}: n \in \mathbf{Z}\right\}$ for which $\Sigma\left\{\nu_{n}: n \in \mathbf{Z}\right\} \leq 1$. We now let $A$ be the Banach space $L^{1}(\mathbf{T})$ but with a new product: if $x$ and $y$ belong to $A$ we now decree their product $x y$ to be the sum of the absolutely convergent series $\sum \nu_{n}\left(x, \phi_{n}\right)\left(y, \phi_{n}\right) \phi_{n}$. It can then be verified that $A$ is a commutative Banach algebra and that the maximal ideal space can be identified once again with $\mathbf{Z}$, but in this instance the multiplicative functional $h_{n}$ is defined by $h_{n}(x)=\nu_{n}\left(x, \phi_{n}\right)$. (See Coddington [1].) The norm of $h_{n}$ is therefore $\nu_{n}$, while the maximal ideal $I_{n}=\operatorname{ker} h_{n}$ is the same as it was in the case of the group algebra, namely $I_{n}=\left\{x \in L^{1}(\mathbf{T}):\left(x, \phi_{n}\right)=0\right\}$. Thus, for all $x \in A$ and $n \in \mathbf{Z}$, we now have

$$
\|\tilde{x}(n)\|=\left\|x+I_{n}\right\|=\left|\left(x, \phi_{n}\right)\right|=\frac{1}{\nu_{n}}\left|h_{n}(x)\right|=\frac{1}{\nu_{n}}|\hat{x}(n)| .
$$

In this case, the identity section is unbounded, since $\left\|\sigma_{e}(n)\right\|=$ (the norm of the identity coset in $\left.A / I_{n}\right)=1 /\left\|h_{n}\right\|=1 / \nu_{n}$. 
2. Bundles of Banach modules. In the first section we considered the representation of a Banach algebra by sections of a bundle of Banach algebras. Now we shall be concerned with the representation of Banach modules by sections of bundles of Banach modules. In the case of a non-commutative algebra it will be necessary to distinguish between left-, right-, and bi-modules over it. For the sake of simplicity we restrict our attention to left-modules and ignore the corresponding results for rightand bi-modules.

Suppose now that $\pi: E \rightarrow S$ is a bundle of Banach algebras and that $\rho: F \rightarrow S$ is a bundle of Banach spaces. Suppose further that for each $p \in S$ the stalk $F_{p}=\rho^{-1}(p)$ is a left Banach module over the stalk $E_{p}=\pi^{-1}(p)$. Thus for a pair $(a, x)$ belonging to the fibered product $E \vee F=\{(b, y) \in E \times F: \rho(b)=\pi(y)\}$ the product $a x$ is defined, and this gives rise to an operation "module multiplication" $\mu: E \vee F \rightarrow F$. We now state an analogue to Proposition 1.1.

Proposition 2.1. Suppose $\pi: E \rightarrow S$ and $\rho: F \rightarrow S$ are as above. Then the following are equivalent:

(1) the map $\mu: E \vee F \rightarrow F$ is continuous;

(2) given any two elements $a_{0} \in E$ and $x_{0} \in F$ for which $\pi\left(a_{0}\right)=\rho\left(x_{0}\right)$ and given any neighborhood W of $a_{0} x_{0}$, there exist neighborhoods $\mathcal{Q}$ and $\mathcal{V}$ of $a_{0}$ and $x_{0}$ respectively such that ax $\in \mathcal{Q} \int$ whenever $a \in \mathcal{Q}, x \in \mathcal{V}$ and $\pi(a)=\rho(x)$;

(3) whenever $\sigma$ is a local section of the bundle $\pi: E \rightarrow S$ and $\tau$ is a local section of the bundle $\rho: F \rightarrow S$, the pointwise product $\sigma \tau$ is a local section of the bundle $\rho: F \rightarrow S$;

(4) there exists a full set $\&$ of local sections of $\pi$ and a full set Th of local sections of $\rho$ such that $\mathfrak{T}$ is closed under left-pointwise multiplication by

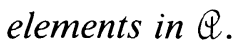

The proof is omitted.

When the above conditions are satisfied we naturally say that $\rho$ : $F \rightarrow S$ is a bundle of left Banach modules over the bundle $\pi: E \rightarrow S$. Given such a bundle it follows that $\Gamma_{b}(\rho)$ is a left Banach module over $\Gamma_{b}(\pi)$.

Suppose that $I$ is a closed two-sided ideal in a Banach algebra $A$ and let $M$ be a left Banach module over $A$. Denote by $I M$ the closed linear span of $\{a x: a \in I, x \in M\}$. Then $I M$ is a closed submodule of $M$. The quotient space $M / I M$ is likewise a left module over $A$, but, more importantly, it is a module over $A / I$. 
Proposition 2.2. Let $A, I$, and $M$ be as above. Then the Banach space $M / I M$ is a left Banach module over the quotient algebra $A / I$ where the action of $A / I$ on $M / I M$ is characterized by the identity

$$
(a+I)(x+I M)=a x+I M
$$

for all $a \in A$ and $x \in M$.

Proof. The problem here is to show that things are well-defined and that the quotient norms behave in the proper way. Here are the straightforward but tedious details.

Given $a \in A$, we define a map $L_{a}: M \rightarrow M / I M$ by

$$
L_{a}(x)=a x+I M .
$$

Clearly $L_{a}$ is linear and $\left\|L_{a}\right\| \leq\|a\|$. If $x \in I M$, then $a x$ also belongs to $I M$ so $L_{a}(x)=0$. Thus, $I M \subset \operatorname{ker} L_{a}$ which means there is a unique linear $\operatorname{map} \tilde{L}_{a}: M / I M \rightarrow M / I M$ such that $\left\|\tilde{L}_{a}\right\|=\left\|L_{a}\right\| \leq\|a\|$ and

$$
\tilde{L}_{a}(x+I M)=L_{a}(x)=a x+I M ;
$$

for all $x \in M$. Consider next the map $\phi: A \rightarrow B(M / I M, M / I M)$ defined by $\phi(a)=\tilde{L}_{a}$. It is easily checked that $\phi$ is linear and since $\|\phi(a)\|=\left\|\tilde{L}_{a}\right\|$ $\leq\|a\|,\|\phi\| \leq 1$. If $a \in I$, then for each $x \in M$, $a x \in I M$ so $L_{a}(x)=0$. Thus $L_{a}=0$ and consequently $\phi(a)=\tilde{L}_{a}=0$. Thus $I \subset \operatorname{Ker} \phi$, which means there is a unique linear map $\tilde{\phi}: A / I \rightarrow B(M / I M, M / I M)$ such that $\|\tilde{\phi}\|=\|\phi\| \leq 1$ and $\tilde{\phi}(a+I)=\phi(a)$ for all $a \in A$.

Given $\alpha \in A / I$ and $\xi \in M / I M$ we now define the product $\alpha \xi$ to be $[\tilde{\phi}(a)](\xi)$. The product $\alpha \xi$ is visibly bilinear and $\|\alpha \xi\| \leq\|\tilde{\phi}(a)\|\|\xi\| \leq$ $\|\tilde{\phi}\|\|\alpha\|\|\xi\| \leq\|\alpha\|\|\xi\|$. Thus, $M / I M$ is a left Banach module over $A / I$. Finally, if $a \in A$ and $x \in M$,

$$
\begin{aligned}
(a+I)(x+I M) & =[\tilde{\phi}(a+I)](x+I M) \\
& =[\phi(a)](x+I M)=\tilde{L}_{a}(x+I M)=L_{a}(x) \\
& =a x+I M .
\end{aligned}
$$

We come now to the main result: if we can represent an algebra by sections of a canonical bundle, then every module over the algebra can be represented likewise.

Theorem 2.3. Let $A$ be a Banach algebra, let $S$ be a topological space, and suppose that $\left\{I_{p}: p \in S\right\}$ is a family of closed two-sided ideals of $A$ which is topologically compatible with $S$. If $M$ is any left Banach module over $A$, then the family of subspaces $\left\{I_{p} M: p \in S\right\}$ of $M$ is also topologically 
compatible with $S$. If we denote by $\pi: E \rightarrow S$ and $\rho: F \rightarrow S$ the canonical bundles of Banach spaces associated with the families $\left\{I_{p}: p \in S\right\}$ and $\left\{I_{p} M: p \in S\right\}$ respectively, then $\rho: F \rightarrow S$ is a bundle of left Banach modules over the bundle $\pi: E \rightarrow S$ of Banach algebras. Moreover, the pair of associated Gelfand morphisms

$$
(\wedge, \wedge):(M, A) \rightarrow\left(\Gamma_{b}(\rho), \Gamma_{b}(\pi)\right)
$$

is a module homomorphism, that is, $\widehat{a x}=\hat{a} \hat{x}$ for all $a \in A$ and $x \in M$.

Proof. We must show that for each $x \in M$ the map $p \rightarrow\left\|x+I_{p} M\right\|$ is upper semicontinuous on $S$ (for that is precisely the definition of compatibility). Suppose, then, that $\left\|x+I_{p} M\right\|<\varepsilon$. We must then show that the inequality $\left\|x+I_{q} M\right\|<\varepsilon$ holds for all points $q$ belonging to some neighborhood $V$ of $p$.

First we may choose elements $a_{1}, \ldots, a_{n}$ in $I_{p}$ and elements $y_{1}, \ldots, y_{n}$ in $M$ such that

$$
\left\|x+I_{p} M\right\| \leq\left\|x+\sum_{k=1}^{n} a_{i} y_{l}\right\|<\varepsilon .
$$

Since $\hat{a}_{t}(p)=0, i=1, \ldots, n$, there is a neighborhood $V$ of $p$ such that for all $q \in V$,

$$
\left\|\hat{a}_{i}(q)\right\|<\left(\varepsilon-\left\|x+\sum_{k=1}^{n} a_{i} y_{l}\right\|\right) / \sum_{l=1}^{n}\left\|y_{i}\right\| ;
$$

$i=1, \ldots, n$. Hence, if $q \in V$,

$$
\begin{aligned}
\left\|x+I_{q} M\right\| & =\inf \left\{\|x+z\|: z \in I_{q} M\right\} \\
& \leq \inf \left\{\left\|x+\sum_{k=1}^{n} a_{i} y_{i}\right\|+\left\|\sum a_{\imath} y_{i}+z\right\|: z \in I_{q} M\right\} \\
& =\left\|x+\sum a_{\imath} y_{i}\right\|+\left\|\sum a_{\imath} y_{i}+I_{q} M\right\| \\
& =\left\|x+\sum a_{\imath} y_{l}\right\|+\left\|\sum\left(a_{\imath}+I_{q}\right)\left(y_{l}+I_{q} M\right)\right\| \\
& \leq\left\|x+\sum a_{\imath} y_{\imath}\right\|+\sum\left\|a_{i}+I_{q}\right\|\left\|y_{\imath}+I_{q} M\right\| \\
& \leq\left\|x+\sum a_{\imath} y_{\imath}\right\|+\sum\left\|\hat{a}_{l}(q)\right\|\left\|y_{l}\right\| \\
& <\left\|x+\sum a_{\imath} y_{\imath}\right\|+\frac{\varepsilon-\left\|x+\sum a_{\imath} y_{i}\right\|}{\sum\left\|y_{i}\right\|} \cdot \sum\left\|y_{l}\right\| \\
& =\varepsilon,
\end{aligned}
$$

which completes the proof of uppersemicontinuity. 
The rest follows from the previous two theorems. To apply the first we observe, first of all that

$$
\widehat{a x}(p)=a x+I_{p} M=\left(a+I_{p}\right)\left(x+I_{p} M\right)=\hat{a}(p) \hat{x}(p)
$$

for all $a \in A, x \in M$, and $p \in S$, thereby establishing the identity $a x=\hat{a} \hat{x}$. We then set $Q=\hat{A}=\{\hat{a}: a \in A\}$ and $\mathscr{K}=\hat{M}=\{\hat{x}: x \in \mathfrak{M}\}$, apply Proposition 2.1, etc.

Corollary 2.4. Let $A$ be a commutative Banach algebra and let $\pi$ : $E \rightarrow \Delta$ be the canonical bundle of Banach algebras associated with the family $\left\{I_{h}: h \in \Delta\right\}$ of regular maximal ideals of $A$. If $M$ is any Banach module over $A$, then there is a bundle of Banach modules $\rho: F \rightarrow \Delta$ over $\pi$ in which the stalk above any point $h \in \Delta$ is (isomorphic to) $M / I_{h} M$. Moreover, the associated morphism ^: $M \rightarrow \Gamma_{b}(\rho)$ which assigns to each $x \in M$ the coset $x+I_{h} M$ is $\sim$-linear, where $\sim: A \rightarrow \Gamma_{b}(\pi)$ is the primitive Gelfand representation of $A$, i.e.

$$
\widehat{a x}=\tilde{a} \hat{x}
$$

for all $a \in A$ and $x \in M$.

Returning to the general situation described in Theorem 2.3, we observe that our representation ${ }^{\wedge}: M \rightarrow \Gamma_{b}(\rho)$ has an important universal property.

Theorem 2.5. (Universal Mapping Property). Let $A, S,\left\{I_{p}: p \in\right.$ $S\}$, and $M$ be as in Theorem 2.3. Suppose that $\phi: M \rightarrow \Gamma_{b}(\xi)$ is a representation of $M$ by sections of a bundle $\xi: G \rightarrow S$ of Banach modules over the canonical bundle of algebras $\pi: E \rightarrow S$; suppose further that $\phi$ is -linear, i.e. that

$$
\phi(a x)=\hat{a} \phi(x)
$$

for all $a \in A, x \in M$. Then there exists a unique bundle map $\tilde{\phi}: \rho \rightarrow \xi$ such that for all $x \in M, \phi(x)=\tilde{\phi} \circ \hat{x} ;$ moreover, $\|\tilde{\phi}\| \leq\|\phi\|$.

The old proof (to Theorem 2.7 in [7]) works. We remark, however, that the pair of bundle maps $\tilde{\phi}: \rho \rightarrow \xi$ and id: $\pi \rightarrow \pi$ is a morphism in the category of bundles of Banach modules over bundles of Banach algebras, i.e. in addition to their being bundle maps the restricted stalk mappings

$$
\left(\tilde{\phi}_{p}, \mathrm{id}_{p}\right):\left(F_{p}, E_{p}\right) \rightarrow\left(G_{p}, E_{p}\right)
$$


are module homomorphisms. We also remark that the universal mapping property for $\wedge: M \rightarrow \Gamma_{b}(\rho)$ characterizes the bundle $\rho: F \rightarrow S$ up to (bundle) isomorphism.

Let us return for a moment to the commutative situation and consider once again the differences between our two Gelfand representations - the usual one and the primitive one. Let $(M, A)$ be a Banach module, where $A$ is commutative. Let us also agree to use the notations established in Corollary 2.4. The module $(M, A)$ is said to have the $(\mathrm{KR})$ condition if for each $h \in \Delta$ we have

$$
\left(M^{*}\right)_{h}=\left(I_{h} M\right)^{\perp}
$$

where $\perp$ denotes the annihilator in $M^{*}$, and $\left(M^{*}\right)_{h}=\left\{F \in M^{*}: F(a x)=\right.$ $\hat{a}(h) F(x)$ for all $x \in M\}$; if $A$ has a bounded approximate identity and $M$ is essential, for instance, then $(M, A)$ will have the (KR) property. (See [7].) In [7] the (KR) condition was used to prove that the family of submodules $\left\{I_{h} M: h \in \Delta\right\}$ is topologically compatible with $\Delta$; from this the existence of the bundle of Banach spaces $\rho: F \rightarrow \Delta$ and the "Gelfand representation" $\wedge: M \rightarrow \Gamma_{b}(\rho)$ then followed. We now know that the (KR) condition was unnecessary for this purpose. The (KR) condition, however, was also used at another point, namely, in showing that the representation ^: $M \rightarrow \Gamma_{b}(\rho)$ is of "Gelfand type," i.e. that it is ^-linear as opposed to $\sim$-linear. So the situation is now the following. We know that in all cases the canonical bundle $\rho: F \rightarrow \Delta$ and the Gelfand representation: ${ }^{\wedge}: M \rightarrow$ $\Gamma_{b}(\rho)$ exist. When we view $\Gamma_{b}(\rho)$ as a module over $\Gamma_{b}(\pi)$ we also know that our representation of $M$ is $\sim$-linear, i.e. $\widehat{a x}=\tilde{a} \hat{x}$ for all $a \in A$ and $x \in M$, or to put it in yet another way, the pair of maps

$$
(\wedge, \sim):(M, A) \rightarrow\left(\Gamma_{b}(\rho), \Gamma_{b}(\pi)\right)
$$

is a homomorphism of modules. In any event, we can also view $\Gamma_{b}(\rho)$ as a module over $C_{0}(\Delta)$ and we can then ask whether our representation of $M$ is -linear.

THEOREM 2.6. Let $M$ and $A$ be as above. The Gelfand representation ^: $M \rightarrow \Gamma_{b}(\rho)$ is of Gelfand type, that is to say, the pair of maps

$$
(\wedge, \wedge):(M, A) \rightarrow\left(\Gamma_{b}(\rho), C_{0}(\Delta)\right)
$$

is a module homomorphism, if and only if the module $(M, A)$ satisfies the (KR) condition.

Proof. In [7] it was shown that the (KR) condition implies that the representation is of Gelfand type. So it suffices now to prove the converse. 
Let us assume, then, that our representation is of Gelfand type, i.e. that $\widehat{a x}=\hat{a} \hat{x}$ for all $a \in A$ and $x \in M$. We must show that for each $h \in \Delta,\left(I_{h} M\right)^{\perp}$, the annihilator of $I_{h} M$ in $M^{*}$, is contained in

$$
\left(M^{*}\right)_{h}=\left\{F \in M^{*}: F(a x)=\hat{a}(h) F(x) \text { for all } a \in A \text { and } x \in M\right\} .
$$

(The last sentence is simply the definition of the (KR) condition.) Thus, assuming that $F \in\left(I_{h} M\right)^{\perp}$, we must show that $F$ belongs to $\left(M^{*}\right)_{h}$, which is to say that

$$
F(a x)=\hat{a}(h) F(x)
$$

for all $a \in A$ and $x \in M$, or equivalently, that

$$
F(a x-\hat{a}(h) x)=0 .
$$

But, by our assumption on ${ }^{\wedge}: M \rightarrow \Gamma_{b}(\rho)$,

$$
(a x-\hat{a}(h) x)^{\wedge}(h)=\hat{a}(h) \hat{x}(h)-\hat{a}(h) \hat{x}(h)=0,
$$

which means precisely that $a x-\hat{a}(h) x$ belongs to $I_{h} M$. Since $F$ is assumed to belong to $\left(I_{h} M\right)^{\perp}$,

$$
F(a x-\hat{a}(h) x)=0 .
$$

So the situation is now quite clear: in working with the Gelfand representation ${ }^{\wedge}: M \rightarrow \Gamma_{b}(\rho)$ the use of the primitive Gelfand representation of $A$ is always appropriate, even when $M$ is non-essential and $A$ lacks even approximate identities, whereas use of the classical Gelfand representation is appropriate precisely when $(M, A)$ satisfies the $(\mathrm{KR})$ condition.

The sectional representations described in Theorem 2.3 have been studied previously in more specialized contexts. In the work of Takahashi ([9] and [10]) and Greene [5], for instance, they are used in the study of Hilbert modules over $C^{*}$-algebras and bundles thereof.

3. Tensor products. This section concerns inductive tensor products of bundles of algebras and bundles of Banach modules. These products are also related to the sectional representations studied in the first two sections of the paper. The reader is referred to [8] for terminology and notations.

The first two theorems concern algebra bundles.

Proposition 3.1. Suppose $\pi: E \rightarrow S$ and $\rho: F \rightarrow T$ are bundles of Banach algebras, where $S$ and $T$ are locally compact Hausdorff. Then the projective tensor product $\pi \hat{\otimes} \rho: E \hat{\otimes} F \rightarrow S \times T$ is also a bundle of Banach 
algebras. Moreover, the natural map

$$
\phi: \Gamma_{0}(\pi) \hat{\otimes} \Gamma_{0}(\rho) \rightarrow \Gamma_{0}(\pi \hat{\otimes} \rho),
$$

which is characterized by the identity $\phi(\sigma \otimes \tau)=\sigma \odot \tau$, is an algebra homomorphism.

Proof. To show that $\pi \hat{\otimes} \rho$ is a bundle of algebras it suffices (by Proposition 1.1) to show that $\Gamma_{0}(\pi \hat{\otimes} \rho)$ is closed under multiplication. To that end we first observe that the set $\left\{\sigma \odot \tau: \sigma \in \Gamma_{0}(\pi), \tau \in \Gamma_{0}(\rho)\right\}$ is closed under multiplication; this is a consequence of the identity

$$
\left(\sigma_{1} \odot \tau_{1}\right)\left(\sigma_{2} \odot \tau_{2}\right)=\left(\sigma_{1} \sigma_{2}\right) \odot\left(\tau_{1} \tau_{2}\right)
$$

and the fact that $\Gamma_{0}(\pi)$ and $\Gamma_{0}(\rho)$ are algebras. If we let $A$ denote the set of all finite sums of sections of the form $\sigma \odot \tau$, then it easily follows that $A$ is also closed under multiplication. Since $A$ is dense in $\Gamma_{0}(\pi \hat{\otimes} \rho)$ (by Corollary 3.5 in [8]), it follows by standard arguments that $\Gamma_{0}(\pi \hat{\otimes} \rho)$ is closed under multiplication.

The proof that $\phi$ is an algebra homomorphism proceeds similarly. For monomials we have

$$
\begin{aligned}
\phi\left(\left(\sigma_{1} \otimes \rho_{1}\right)\left(\sigma_{2} \otimes \tau_{2}\right)\right) & =\phi\left(\left(\sigma_{1} \sigma_{2}\right) \otimes\left(\tau_{1} \tau_{2}\right)\right) \\
& =\left(\sigma_{1} \sigma_{2}\right) \odot\left(\tau_{1} \tau_{2}\right)=\left(\sigma_{1} \odot \tau_{1}\right)\left(\sigma_{2} \odot \tau_{2}\right) \\
& =\phi\left(\sigma_{1} \odot \tau_{1}\right) \phi\left(\sigma_{2} \odot \tau_{2}\right) .
\end{aligned}
$$

Since $\phi$ is linear and continuous, it follows that $\phi(\xi \eta)=\phi(\xi) \phi(\eta)$ holds for all $\xi$ and $\eta$ in $\Gamma_{0}(\pi) \hat{\otimes} \Gamma_{0}(\rho)$.

If $A$ and $B$ are commutative Banach algebras with maximal ideal spaces $S$ and $T$ respectively, then the maximal ideal space of $A \hat{\otimes} B$ can be identified with $S \times T$ in such a way that we have

$$
(a \otimes b)^{\wedge}(s, t)=\hat{a}(s) \hat{b}(t)=(\hat{a} \odot \hat{b})(s, t)
$$

for all $a \in A, b \in B, s \in S, t \in T$. We shall now prove an analogous result.

THEOREM 3.2. Suppose the following are given:

(a) $A$ and $B$ are Banach algebras,

(b) $S$ and $T$ are compact Hausdorff spaces;

(c) $\left\{I_{s}: s \in S\right\}$ is a family of closed two-sided ideals in A; the family is topologically compatible with $S$;

(d) $\left\{J_{t}: t \in T\right\}$ is a family of closed two-sided ideals in $B$; the family is topologically compatible with $T$. 
Denote by $\pi: E \rightarrow S$ and $\rho: F \rightarrow T$ the corresponding canonical bundles of Banach algebras and by ${ }^{\wedge}: A \rightarrow \Gamma(\pi)$ and ${ }^{\wedge}: B \rightarrow \Gamma(\rho)$ the corresponding Gelfand morphisms.

Then there exists a family of closed two-sided ideals $\left\{I_{s t}: s \in S, t \in T\right\}$ in $A \hat{\otimes} B$ such that

(1) the family $\left\{I_{s t}:(s, t) \in S \times T\right\}$ is topologically compatible with $S \times T$;

(2) the corresponding canonical bundle of Banach algebras can be naturally identified with the tensor product bundle $\pi \hat{\otimes} \rho: E \hat{\otimes} F \rightarrow S \times T$;

(3) so identified, the corresponding Gelfand morphism ^ : $A \hat{\otimes} B \rightarrow$ $\Gamma(\pi \hat{\otimes} \rho)$ has the following property:

$$
(a \otimes b)^{\wedge}=\hat{a} \odot \hat{b}
$$

for all $a \in A$ and $b \in B$.

Proof. There is a natural norm-decreasing linear map $\theta: A \hat{\otimes} B \rightarrow$ $\Gamma(\pi \hat{\otimes} \rho)$ such that

$$
\theta(a \otimes b)=\hat{a} \odot \hat{b}
$$

for all $a \in A, b \in B$, namely, $\theta$ is the composition of the tensor product of the Gelfand morphisms $\wedge \wedge$ : $A \hat{\otimes} B \rightarrow \Gamma(\pi) \hat{\otimes} \Gamma(\rho)$ with the natural map $\phi: \Gamma(\pi) \hat{\otimes} \Gamma(\rho) \rightarrow \Gamma(\pi \hat{\otimes} \rho)$ described in Proposition 3.1. Since $\wedge \otimes$. and $\phi$ are both algebra homomorphisms, so is $\theta$. Given $(s, t) \in S \times T$ we denote by $\mathrm{ev}_{s t}: \Gamma(\pi \hat{\otimes} \rho) \rightarrow E_{s} \hat{\otimes} F_{t}$ the map which assigns to each section its value at the point $(s, t)$. Then $\mathrm{ev}_{s t}$ is also a norm-decreasing algebra homomorphism. We define $I_{s t}$ to be the kernel of the homomorphism $\mathrm{ev}_{s t} \circ \theta: A \hat{\otimes} B \rightarrow E_{s} \hat{\otimes} F_{t}$.

We will now show that the family $\left\{I_{s t}\right\}$ has the properties advertised above. Clearly each $I_{s t}$ is a closed two-sided ideal. Let us denote by $\phi_{s t}$ : $(A \hat{\otimes} B) / I_{s t} \rightarrow E_{s} \hat{\otimes} F_{t}$ the norm-decreasing homomorphism induced by $\mathrm{ev}_{s t} \circ \theta$; thus, for all $a \in A$ and $b \in B$,

$$
\phi_{s t}\left(a \hat{\otimes} b+I_{s t}\right)=\hat{a}(s) \otimes \hat{b}(t) .
$$

We will show that the map $\phi_{s t}:(A \hat{\otimes} B) / I_{s t} \rightarrow E_{s} \hat{\otimes} F_{t}$ is an isometric isomorphism.

We define a map $\psi: A \times B \rightarrow(A \hat{\otimes} B) / I_{s t}$ by setting $\psi(a, b)=a \otimes b$ $+I_{s t}$. This map is clearly bilinear with norm less than or equal to one. If $a \in I_{s}$ and $b \in B$, then $\hat{a}(s)=0$ so

$$
\left(\mathrm{ev}_{s t} \circ \theta\right)(a \otimes b)=\hat{a}(s) \otimes \hat{b}(t)=0
$$

which means $(a \otimes b) \in I_{s t}$ and thus $\psi(a, b)=0$. Similarly $\psi(a, b)=0$ for all $a \in A, b \in J_{t}$. Consequently (by Lemma 1.2 of [8]), there is an 
induced bilinear map $\bar{\psi}: E_{s} \times F_{t}=\left(A / I_{s}\right) \times\left(B / J_{J}\right) \rightarrow(A \hat{\otimes} B) / I_{s t}$ with $\|\bar{\psi}\|=\|\psi\| \leq 1$ such that

$$
\bar{\psi}(\hat{a}(s), \hat{b}(t))=\psi(a, b)=a \otimes b+I_{s t} .
$$

Finally, we get a linear map $\tilde{\psi}: E_{s} \hat{\otimes} F_{t} \rightarrow(A \hat{\otimes} B) / I_{s t}$ such that $\|\tilde{\psi}\|=$ $\|\bar{\psi}\| \leq 1$ and

$$
\tilde{\psi}(\hat{a}(s) \otimes \hat{b}(t))=\bar{\psi}(\hat{a}(s), \hat{b}(t))=a \otimes b+I_{s t}
$$

for all $a \in A, b \in B$. It is easily argued that $\tilde{\psi} \circ \phi_{s t}$ and $\phi_{s t} \circ \tilde{\psi}$ are the identity maps on $(A \hat{\otimes} B) / I_{s t}$ and $E_{s} \hat{\otimes} F_{t}$ respectively, which shows, of course, that the maps $\phi_{s t}$ and $\tilde{\psi}$ are bijective and inverse to one-another. Finally, since both maps are norm-decreasing, both maps must, in fact, be norm-preserving.

From the fact that the maps $\phi_{s t}:(A \hat{\otimes} B) / I_{s t} \rightarrow E_{s} \hat{\otimes} F_{t}$ are isometries, it follows that the family $\left\{I_{s t}\right\}$ is topologically compatible with $S \times T$. For, if $z \in A \hat{\otimes} B$, then

$$
\left\|z+I_{s t}\right\|=\left\|\phi_{s t}\left(z+I_{s t}\right)\right\|=\|\theta(z)(s, t)\|,
$$

and since $\theta(z)$ is a section of the bundle $\pi \hat{\otimes} \rho$ the map $(s, t) \rightarrow$ $\|\theta(z)(s, t)\|=\left\|z+I_{s t}\right\|$ must be uppper semicontinuous on $S \times T$.

If we identify the stalk $(A \hat{\otimes} B) / I_{s t}$ in the canonical bundle with $E_{s} \hat{\otimes} F_{t}$ (via the natural map $\phi_{s t}$ ), then the Gelfand morphism $\wedge: A \hat{\otimes} B \rightarrow$ $\Gamma(\pi \hat{\otimes} \rho)$ has the desired property:

$$
\begin{aligned}
& (a \otimes b) \wedge(s, t)=a \otimes b+I_{s t} \\
& \quad \text { is identified with } \phi_{s t}(a \otimes b)=\hat{a}(s) \otimes \hat{b}(t) .
\end{aligned}
$$

In the remainder of the paper we shall consider tensor products of bundles of modules.

Proposition 3.3. Suppose $\pi: E \rightarrow S$ and $\rho: F \rightarrow T$ are bundles of Banach algebras where $S$ and $T$ are locally compact Hausdorff. Suppose $\pi^{\prime}$ : $E^{\prime} \rightarrow S$ and $\rho^{\prime}: F^{\prime} \rightarrow T$ are bundles of Banach modules over the algebra bundles. Then $\pi^{\prime} \hat{\otimes} \rho^{\prime}: E^{\prime} \hat{\otimes} F^{\prime} \rightarrow S \times T$ is a bundle of Banach modules over the bundle of Banach algebras $\pi \hat{\otimes} \rho: E \hat{\otimes} F \rightarrow S \times T$. The natural map

$$
\begin{aligned}
(\phi, \phi): & \left(\Gamma_{0}\left(\pi^{\prime}\right) \hat{\otimes} \Gamma_{0}\left(\rho^{\prime}\right), \Gamma_{0}(\pi) \hat{\otimes} \Gamma_{0}(\rho)\right) \\
\rightarrow & \left(\Gamma_{0}\left(\pi^{\prime} \hat{\otimes} \rho^{\prime}\right), \Gamma_{0}(\pi \hat{\otimes} \rho)\right)
\end{aligned}
$$

characterized by $\phi(\sigma \otimes \tau)=\sigma \odot \tau$, is a module homomorphism. 
The proof, which is analogous to the proof of Proposition 3.1, is omitted.

Theorem 3.4. Suppose we are given the data of Theorem 3.2. Suppose additionally that $(M, A)$ and $(N, B)$ are essential modules. We denote by $\pi^{\prime}$ : $E^{\prime} \rightarrow S$ and $\rho^{\prime}: F^{\prime} \rightarrow T$ the canonical bundles corresponding to the families $\left\{I_{s} M: s \in S\right\}$ and $\left\{J_{t} N: t \in T\right\}$ respectively and we denote by ${ }^{\wedge}: M \rightarrow \Gamma\left(\pi^{\prime}\right)$ and $\wedge: N \rightarrow \Gamma\left(\rho^{\prime}\right)$ the Gelfand morphisms. Then the canonical bundle corresponding to the family $\left\{I_{s t}(M \hat{\otimes} N):(s, t) \in S \times T\right\}$ can be identified with the product bundle $\pi^{\prime} \hat{\otimes} \rho^{\prime}: E^{\prime} \hat{\otimes} F^{\prime} \rightarrow S \times T$ in such a way that the Gelfand morphism ${ }^{\wedge}: M \hat{\otimes} N \rightarrow \Gamma\left(\pi^{\prime} \hat{\otimes} \rho^{\prime}\right)$ has the following property:

$$
(x \otimes y)^{\wedge}=\hat{x} \odot \hat{y}
$$

for all $x \in M$ and $y \in N$.

The reader will find that this theorem can be proved by modifying only slightly the proof of Proposition 1.3 in [8].

THEOREM 3.5. Let $A$ be a Banach algebra and let $S$ be a compact Hausdorff space. Suppose $\left\{I_{s}: S \in S\right\}$ is a family of closed two-sided ideals in $A$, the family being topologically compatible with $S$. We denote by $\xi$ : $\mathbb{Q} \rightarrow S$ the corresponding canonical bundle of Banach algebras.

Suppose $M$ and $N$ are A-modules. We denote by $\pi: E \rightarrow S, \rho: F \rightarrow S$, and $\theta: H \rightarrow S$ the bundles of Banach modules over $\xi: \mathbb{Q} \rightarrow S$ which correspond to the A-modules $M, N$, and $M \hat{\otimes}_{A} N$ respectively, and we denote all Gelfand morphisms by ^.

Then for each $s \in S$ there exists a unique linear isometry $\phi_{s}: H_{s} \rightarrow$ $E_{\mathrm{s}} \hat{\otimes}_{\mathbb{Q}_{s}} F_{\mathrm{s}}$ such that

$$
\phi_{s}\left(\left(x \otimes_{A} y\right)^{\wedge}(s)\right)=\hat{x}(s) \otimes_{\mathbb{Q},} \hat{y}(s) .
$$

Proof. We define a map $f: M \times N \rightarrow H_{s}$ by

$$
f(x, y)=\left(x \otimes_{A} y\right)^{\wedge}(s) .
$$

Then $f$ is clearly an $A$-balanced bilinear map and $\|f\| \leq 1$. Moreover, for all $a \in A, x \in M$, and $y \in N$

$$
\begin{aligned}
f(a x, y) & =\left(a x \otimes_{A} y\right)^{\wedge}(s)=\left(a\left(x \otimes_{A} y\right)\right)^{\wedge}(s) \\
& =\hat{a}(s)(x \otimes y) \wedge(s)=\hat{a}(s) f(x, y) .
\end{aligned}
$$


Thus $f(a x, y)=0$, if $a \in I_{s}$. Similarly $f(x, a y)=f(a x, y)=0$ if $a \in I_{s}$. It follows that there is a unique bilinear map

$$
\tilde{f}: E_{s} \times F_{s}=\left(M / I_{s} M\right) \times\left(N / I_{s} N\right) \rightarrow H_{s}
$$

such that

$$
\begin{aligned}
\tilde{f}(\hat{x}(s), \hat{y}(s)) & =\tilde{f}\left(x+I_{s} M, y+I_{s} N\right) \\
& =f(x, y)=\left(x \otimes_{A} y\right)^{\wedge}(s) .
\end{aligned}
$$

Moreover, $\|\tilde{f}\|=\|f\| \leq 1$. Suppose $\alpha \in \mathbb{Q}_{s}$, say $\alpha=\hat{a}(s)$ for some $a \in A$. Then

$$
\begin{aligned}
\tilde{f}(\alpha \hat{x}(s), \hat{y}(s)) & =\tilde{f}(\widehat{a x}(s), \hat{y}(s))=f(a x, y) \\
& =f(x, a y)=\tilde{f}(\hat{x}(s), \alpha \hat{y}(s)) .
\end{aligned}
$$

Thus, $\tilde{f}$ is $\mathbb{Q}_{s}$-balanced. Therefore, there is a unique linear map $\psi: E_{s} \hat{\otimes}_{\mathbb{Q}_{s}} F_{s}$ $\rightarrow H_{s}$ such that

$$
\psi\left(\hat{x}(s) \otimes_{\mathbb{Q}_{s}} \hat{y}(s)\right)=\left(x \otimes_{A} y\right)^{\wedge}(s) .
$$

Moreover $\|\psi\|=\|\tilde{f}\| \leq 1$.

Next we define a map $g: M \times N \rightarrow E_{s} \otimes_{\mathbb{Q}_{s}} F_{s}$ by setting

$$
g(x, y)=\hat{x}(s) \otimes_{\mathbb{Q}_{s}} \hat{y}(s) .
$$

Then $g$ is bilinear and $\|g\| \leq 1$. Now

$$
\begin{aligned}
g(a x, y) & =\widehat{a x}(s) \otimes_{\mathbb{Q}_{s}} \hat{y}(s)=[\hat{a}(s) \hat{x}(s)] \otimes_{\mathbb{Q}_{s}} \hat{y}(s) \\
& =\hat{x}(s) \otimes_{\mathbb{Q}_{s}} \widehat{a y}(s)=g(x, a y),
\end{aligned}
$$

i.e. $g$ is $A$-balanced. Hence there is a unique linear map $\tilde{g}: M \hat{\otimes}_{A} N \rightarrow$ $E_{s} \otimes_{\mathbb{Q}_{s}} F_{s}$ with

$$
\tilde{g}\left(x \otimes_{A} y\right)=\hat{x}(s) \otimes_{\mathbb{Q}_{3}} \hat{y}(s)
$$

and $\|\tilde{g}\|=\|g\| \leq 1$. Now

$$
\begin{aligned}
\tilde{g}\left(a\left(x \otimes_{A} y\right)\right) & =\tilde{g}\left(a x \otimes_{A} y\right)=\widehat{a x}(s) \otimes_{\mathbb{Q}_{s}} \hat{y}(s) \\
& =[\hat{a}(s) \hat{x}(s)] \otimes_{\mathbb{Q}_{s}} \hat{y}(s)=\hat{a}(s)\left[\hat{x}(s) \otimes_{\mathbb{Q}} \hat{y}(s)\right]
\end{aligned}
$$

which shows that $\tilde{g}\left(a\left(x \otimes_{A} y\right)\right)=0$ if $a \in I_{s}$. It follows that $\tilde{g}$ is zero on $I_{s}\left(M \otimes_{A} N\right)$. It follows that there is a unique linear map $\phi_{s}: H_{s}=$ $\left(M \hat{\otimes}_{A} N\right) / I_{s}\left(M \hat{\otimes}_{A} N\right) \rightarrow E_{s} \otimes_{\mathbb{Q}_{s}} F_{s}$ such that

$$
\begin{aligned}
\phi_{s}\left(\left(x \otimes_{A} y\right)^{\hat{n}}(s)\right) & =\phi_{s}\left(\left(x \otimes_{A} y\right)+I_{s}\left(M \hat{\otimes}_{A} N\right)\right) \\
& =\tilde{g}\left(x \otimes_{A} y\right)=\hat{x}(s) \otimes_{\mathbb{Q}_{s}} \hat{y}(s) .
\end{aligned}
$$

Also $\left\|\phi_{s}\right\|=\|\tilde{g}\| \leq 1$. 
The rest is routine. One shows that $\psi \circ \phi_{s}$ and $\phi_{s} \circ \psi$ are the identity maps on $H_{s}$ and $E_{s} \otimes_{\mathbb{Q}_{s}} F_{s}$ respectively. It follows that the maps $\phi_{s}$ and $\psi$ are bijective and that each is the inverse of the other. Finally, since $\phi_{s}$ and $\psi$ and both norm-decreasing, both must, in fact, be isometries.

Suppose $\xi: \mathbb{Q} \rightarrow S$ is a bundle of Banach algebras and let us suppose that each fiber algebra $\mathcal{Q}_{p}$ has an identity element $e_{p}$. If, additionally, the identity selection $\sigma_{e}$, defined by $\sigma_{e}(p)=e_{p}$, is a section of the bundle $\sigma$, then we shall say that $\xi: \mathbb{Q} \rightarrow S$ is a bundle of Banach algebras with identity.

THEOREM 3.6. Let $S$ be a compact Hausdorff space. Suppose $\xi: \mathbb{Q} \rightarrow S$ is a bundle of Banach algebras with identity and suppose $\pi: E \rightarrow S$ and $\rho$ : $F \rightarrow S$ are bundles of Banach modules over the bundle of algebras. Then there exists a bundle of Banach spaces $\phi: G \rightarrow S$ with the following properties:

(1) $\phi: G \rightarrow S$ is a bundle of Banach modules over the algebra bundle $\xi$ : $\mathbb{Q} \rightarrow S$;

(2) for each $s \in S$ the fiber $G_{s}$ above $s$ is $E_{s} \otimes_{Q_{s}} F_{s}$;

(3) the tensor map $\otimes_{\mathbb{Q}}: E \vee F \rightarrow G$ which assigns to each pair $(x, y) \in$ $E \vee F($ meaning $\pi(x)=\rho(y))$ its product $x \otimes_{\mathbb{Q}_{\pi(2)}} y$ in the stalk $G_{\pi(x)}$, is continuous;

(4) if $\sigma$ and $\tau$ are local sections of the bundles $\pi: E \rightarrow S$ and $\rho: F \rightarrow S$ respectively, then their pointwise $Q_{-}$product $\sigma \odot_{\mathfrak{Q}} \tau$, defined by $\left(\sigma \odot_{\mathfrak{Q}} \tau\right)(s)=$ $\sigma(s) \otimes_{\mathbb{Q}_{s}} \tau(s)$, is a local section of the bundle $\phi: G \rightarrow S$.

Proof. To construct the bundle $\phi: G \rightarrow S$ we apply the previous theorem. We let $A=\Gamma(\xi)$ and for each $s \in S$ we let $I_{s}=\{\sigma \in A$ : $\sigma(s)=0\}$. Then $A$ is a Banach algebra with identity and $\left\{I_{s}: s \in S\right\}$ is a family of closed two-sided ideals in $A$. Moreover, the family is topologically compatible with $S$ and the corresponding canonical bundle of algebras can be identified with the given bundle $\xi: \mathbb{Q} \rightarrow S$ in such a way that the Gelfand morphism ^ ${ }^{\wedge} A \rightarrow \Gamma(\xi)$ is simply the identity map. (See [7], Theorem 4.1.)

We next set $M=\Gamma(\pi)$ and $N=\Gamma(\rho)$ so that $M$ and $N$ are $A$-modules. For each $s \in S$ it is easy to show that

$$
I_{s} M=\{\sigma \in M: \sigma(s)=0\} .
$$

It follows that the canonical bundle associated with the family $\left\{I_{s}: s \in S\right\}$ can be identified with the given bundle $\pi: E \rightarrow S$ in such a way that the Gelfand morphism ${ }^{\wedge}: M \rightarrow \Gamma(\rho)$ is, once again, the identity map. Similar remarks hold for $N$. 
If we denote by $\theta: H \rightarrow S$ the canonical bundle for the $A$-module $M \hat{\otimes}_{A} N=\Gamma(\pi) \hat{\otimes}_{\Gamma(\xi)} \Gamma(\rho)$, then for each $s \in S$, the previous theorem assures us of the existence of a unique isometric isomorphism $\phi_{s}: E_{s} \otimes_{\mathbb{Q}_{s}} F_{s}$ $\rightarrow H_{s}$ such that

$$
\phi_{s}\left(\sigma(s) \otimes_{\mathbb{Q}_{s}} \tau(s)\right)=\phi_{s}\left(\hat{\sigma}(s) \otimes_{\mathbb{Q}_{s}} \hat{\tau}(s)\right)=\left(\sigma \otimes_{A} \tau\right)^{\wedge}(s) ;
$$

for all $\sigma \in M=\Gamma(\pi)$ and $\tau \in N=\Gamma(\rho)$. If we now let $G$ be the disjoint union of the family of Banach modules $\left\{E_{s} \hat{\otimes}_{\mathbb{Q}_{s}} F_{s}: s \in S\right\}$, then we have a bijection $\phi: G \rightarrow H$ whose restrictions to stalks are the maps $\phi_{s}$. We topologize $G$ by transplanting the topology from $H$ to $G$ via the map $\phi^{-1}$, etc.

The remainder of the proof can be modeled after the proof of Theorem 21 in [8].

By analogy with previous notation the bundle $\phi: G \rightarrow S$ might reasonably be denoted by $\pi \hat{\otimes}_{\xi} \rho: E \hat{\otimes}_{\mathfrak{Q}} F \rightarrow S$. If we then return to the situation described in Theorem 3.5, the content of the theorem can now be paraphrased as follows:

Suppose that the data and notations of Theorem 3.5 are given, and suppose also that $A$ has an identity. Then the canonical bundle associated with the module $\left(M \hat{\otimes}_{A} N, A\right)$ can be identified with the product bundle $\pi \hat{\otimes}_{\xi} \rho: E \hat{\otimes}_{\mathfrak{Q}} F \rightarrow S$ in such a way that

$$
\left(x \otimes_{A} y\right)^{\wedge}=\hat{x} \odot_{\mathfrak{Q}} \hat{y}
$$

for all $x \in M, y \in N$.

\section{REFERENCES}

[1] E. Coddington, Some Banach algebras, Proc. Amer. Math. Soc. 8 (1957), 258-261.

[2] M. J. Dupré, The classification and structure of $C^{*}$-algebra bundles, Mem. Amer. Math. Soc., No. 222 (1979).

[3] Duality for $C^{*}$-algebras, Mathematical foundations of quantum theory (Proc. Conf. Loyola Univ., New Orleans, La.) 329-338. Academic Press, New York, 1978.

[4] J. M. G. Fell, The structure of algebras of operator fields, Acta Math., 106 (1961), 233-280.

[5] W. A. Greene, Ambrose modules, Mem. Amer. Math. Soc., No. 148 (1974), 109-133.

[6] K. H. Hofmann, Sheaves and bundles of Banach spaces, (preprint).

[7] J. W. Kitchen and D. A. Robbins, Gelfand representation of Banach modules, Dissertationes Math. (Rozprawy Mat.) (to appear).

[8] _ Tensor products of Banach bundles, Pacific J. Math., 94 (1981), 151-169. 
[9] A. Takahashi, Hilbert modules and their representation, Rev. Colombiana Mat., 13 (1979), 1-38.

[10] A A duality betwen Hilbert modules and fields of Hilbert spaces, Rev. Colombiana Mat., 13 (1979), 93-120.

Received September 10, 1981 and in revised form March 4, 1982.

DUKE UNIVERSITY

DURHAM, NC 27706

AND

TRINITy COLLEGE

HARTFORD, CT 06106 


\section{PACIFIC JOURNAL OF MATHEMATICS}

EDITORS

Donald BABBITt (Managing Editor)

University of California

Los Angeles, CA 90024

Hugo Rossi

University of Utah

Salt Lake City, UT 84112

C. C. Moore and Arthur Ogus

University of California

Berkeley, CA 94720

\section{J. DugundiI}

Department of Mathematics

University of Southern California

Los Angeles, CA 90089-1113

R. Finn and H. SAMELSON

Stanford University

Stanford, CA 94305

\section{ASSOCIATE EDITORS}
R. ARENS
E. F. BECKENBACH
B. H. NEUMANN
F. WOLF
K. YOSHIDA (1906-1982)

\section{SUPPORTING INSTITUTIONS}

UNIVERSITY OF ARIZONA

UNIVERSITY OF BRITISH COLUMBIA

CALIFORNIA INSTITUTE OF TECHNOLOGY

UNIVERSITY OF CALIFORNIA

MONTANA STATE UNIVERSITY

UNIVERSITY OF NEVADA. RENO

NEW MEXICO STATE UNIVERSITY

OREGON STATE UNIVERSITY
UNIVERSITY OF OREGION

UNIVERSITY OF SOUTHERN CALIFORNIA

STANFORD UNIVERSITY

UNIVERSITY OF HAWAII

UNIVERSITY OF TOKYO

UNIVERSITY OF UTAH

WASHINGTON STATE UNIVERSITY

UNIVERSITY OF WASHINGTON 


\section{Pacific Journal of Mathematics}

Vol. 109, No. $1 \quad$ May, 1983

Donald George Babbitt and V. S. Varadarajan, Formal reduction theory of meromorphic differential equations: a group theoretic view $\ldots \ldots \ldots \ldots 1$

Jo-Ann Deborah Cohen, Norms on $F(X) \ldots \ldots \ldots \ldots \ldots \ldots \ldots \ldots \ldots \ldots$

Robert Fitzgerald, Witt kernels of function field extensions $\ldots \ldots \ldots \ldots \ldots 89$

Hervé Jacquet and Joseph Andrew Shalika, The Whittaker models of induced representations .............................. 107

Masakiti Kinukawa, Some generalizations of contraction theorems for

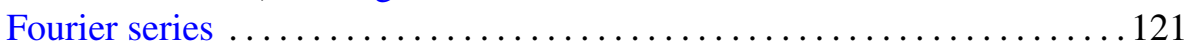

Joseph Weston Kitchen, Jr. and David A. Robbins, Sectional

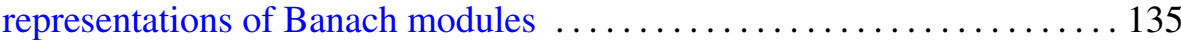

Victor Charles Pestien, Jr., Weak approximation of strategies in measurable gambling

Richard Scott Pierce and Charles Irvin Vinsonhaler, Realizing central

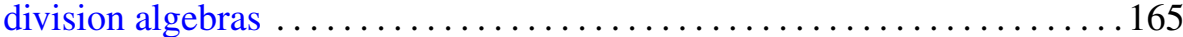

Walter Ricardo Ferrer Santos, Cohomology of comodules ............ 179

Marko Tadić, Harmonic analysis of spherical functions on reductive groups

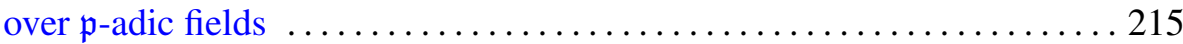

Lorenzo Traldi, The determinantal ideals of link modules. II . . . . . . . . 237

Alain J. Valette, A remark on the Kasparov groups $\operatorname{Ext}^{i}(A, B) \ldots \ldots \ldots 247$ 